\title{
Acute and late coronary outcomes in 1073 patients with Kawasaki disease with and without intravenous $\gamma$-immunoglobulin therapy
}

\author{
Ming-Tai Lin, ${ }^{1}$ Li-Chuan Sun, ${ }^{2}$ En-Ting Wu, ${ }^{1}$ Jou-Kou Wang, ${ }^{1}$ Hung-Chi Lue, ${ }^{1}$ \\ Mei-Hwan Wu ${ }^{1}$
}

${ }^{1}$ Department of Pediatrics, National Taiwan University Hospital and Medical College, National Taiwan University, Taipei, Taiwan

${ }^{2}$ Department of Pediatrics, Cardinal Tien General Hospital, Taipei, Taiwan

\section{Correspondence to} Dr Mei-Hwan Wu, Department of Pediatrics, National Taiwan University Hospital, No. 7, Chung-Shan South Road, Taipei 100, Taiwan;

wumh@ntu.edu.tw

Received 17 March 2014 Revised 23 November 2014 Accepted 10 December 2014 Published Online First 6 January 2015
CrossMark

To cite: Lin M-T, Sun L-C, Wu E-T, et al. Arch Dis Child 2015; 100:542-547.

\section{ABSTRACT}

Objective To explore acute and late coronary outcomes and their risk/modifiers in patients with Kawasaki disease (KD).

Design Retrospective study.

Setting and patients 1073 patients with KD identified from a tertiary care medical centre (19802012; 8677 patient-years).

Main outcome measures The acute coronary severities and late outcomes (survival free of coronary aneurysm persistence and ischaemia) were assessed. Results Coronary arterial lesions occurred in $40.6 \%$ of cases at their acute febrile stages, and persisted beyond 1 month in $196(18.3 \%, M / F=138 / 58)$ patients: 125 (11.6\%) had small aneurysms, 44 (4.1\%) had medium aneurysms, and $27(2.5 \%)$ had giant aneurysms. At follow-up (1-46 years), coronary aneurysms persisted in all with giant aneurysms, in 55\% of those with medium aneurysms (18\% with stenosis), and in $9 \%$ of those with small aneurysms. Ischaemia events occurred in 14 patients $(M / F=13 / 1)$ and caused four deaths. Among the patients with KD with coronary aneurysms, 10-year ischaemia event-free and aneurysm persistence probability was $87.5 \%$ and $20.6 \%$, respectively. The only independent risk for aneurysm persistence was the aneurysm severity 1 month after KD onset $\left(\chi^{2}=80.73, p<10^{-3}\right)$. Male patients and intravenous $\gamma$-immunoglobulin (IVIG) therapy were independent risk factors of initial coronary severity but were not associated with the late coronary outcomes, even in severity stratified subgroups.

Conclusions The coronary severity 1 month after KD onset is most crucial to the late coronary outcomes. Although IVIG use improves the initial severity of coronary lesions, it does not further modify the longterm fate of coronary aneurysms.

\section{INTRODUCTION}

Since the first report in 1967 from Japan, Kawasaki disease (KD) has become the most common form of paediatric systemic vasculitis. ${ }^{1}$ Despite receiving intravenous immunoglobulin (IVIG) therapy, coronary arterial lesions (CALs) occurred in $5-20 \%$ of patients with KD during the acute stage. ${ }^{2-4} \mathrm{CAL}$ may persist and even progress to stenosis or obstruction. Therefore, CAL following KD is now the leading cause of acquired heart disease in children. $^{3-7}$ In our previous study focusing on the period before the introduction of IVIG therapy, CAL occurred in $44 \%$ of patients. ${ }^{8}$ Among them, most of the dilation regressed and all giant

\section{What is already known on this topic}

- Kawasaki disease is one of the most common pediatric systemic vasculitis.

- With intravenous immunoglobulin therapy, the risk of coronary arterial lesions for patients with Kawasaki disease has been reduced from 20 $25 \%$ to $5-10 \%$ during the acute stage. However, the late coronary profiles and their risk factors are still unclear.

\section{What this study adds}

- The only independent risk factor for aneurysm persistence or ischaemia events was aneurysm severity 1 month after Kawasaki disease onset.

- Male patients and intravenous immunoglobulin therapy were both independent risk factors of initial coronary severity but were not associated with the late coronary outcomes, even in severity stratified subgroups.

aneurysms persisted. IVIG therapy became available in 1986 , but only became standard treatment after the implementation of Taiwan's National Health Insurance programme in mid-1995, which covers the expense. ${ }^{79}$ The relationships between late KD coronary outcomes and the risk/modifiers, like gender and IVIG use, have never been studied as yet. We therefore conducted a longitudinal study based on patient enrolment since 1980 to delineate this issue.

\section{METHODS}

The study was approved by our Institutional Research Board.

\section{Study population}

The diagnosis of KD in this study was made based on clinical criteria for KD. ${ }^{10}$ All children received echocardiography during the febrile stage and the subacute phase $(1,3,6$ weeks, and 3 months after fever onset, and the subsequent frequency varied depending on the severity of the CAL), except patients diagnosed before 1984. CALs were defined as previous studies. ${ }^{411}$ However, only cases where CAL persisted for more than a month after disease onset were considered as coronary aneurysms. 
Based on the aneurysm size, the severity of coronary aneurysms was classified as small $(\leq 4 \mathrm{~mm})$, medium $(4-8 \mathrm{~mm})$ and giant $(>8 \mathrm{~mm}){ }^{4}$ CALs and the regression were diagnosed based on two-dimensional (2D) echocardiography and confirmed using CT or angiography if indicated (when 2D echocardiograms showed regression of coronary aneurysms that had been greater than $4 \mathrm{~mm}$, or for the patients who exhibited symptoms of myocardial ischaemia, or for a regular follow-up of giant aneurysms every 3-5 years, figure 1). For patients with echocardiography follow-up, regression was defined when echocardiography demonstrated coronary arteries with normal appearance and size, as well as normal cardiac function. Low-dose aspirin was given for at least 6-8 weeks and until when echocardiography confirmed the regression of coronary lesions. ECG was performed annually and whenever patients reported chest tightness. Diagnosis of myocardial ischaemia was made based on ST segment changes and/or stress radionuclear imaging results (figure 1).

\section{Statistical methods}

We performed analyses using SPSS V.11.5. Data were expressed as mean \pm SD (medians with ranges), and we used Mann-Whitney test for two-group comparisons. The $\chi^{2}$ test was used to compare categorical data. For the time-related events and outcome variables, like persistence of coronary aneurysm, we used Kaplan-Meier estimates to draw event-free curves and tested their associations with candidate-independent factors by using log-rank tests. To estimate regression probability, the follow-up period ended when the CAL regressed or patients did not attend the scheduled follow-ups. Variables related to time-related events with $\mathrm{p}<0.1$ in the univariable analysis were entered into the multivariate model. Statistical significance was set at $\mathrm{p}<0.05$.

\section{RESULTS}

Patient characteristics and coronary severity 1 month after KD onset

Between 1980 and 2012, 1073 patients were diagnosed with $\mathrm{KD}(\mathrm{M} / \mathrm{F}$ ratio $=664 / 409,1.62)$, including 22 with atypical KD with CAL. The age at diagnosis ranged from 1 month to 18 years (median 1.59 years). The proportions of patients with infancy onset $(28 \%$ vs $34 \%, \mathrm{p}=0.09)$ and male $(61 \%$ vs $63 \%$, $\mathrm{p}=0.46$ ) in patients without and with IVIG were not statistically different. IVIG therapy was not administered in 366 patients (34.1\%). Our national health insurance was implemented in
Figure 1 Flow chart of management and follow-up of 1073 patients with Kawasaki disease (KD) (IVIG, intravenous immunoglobulin).

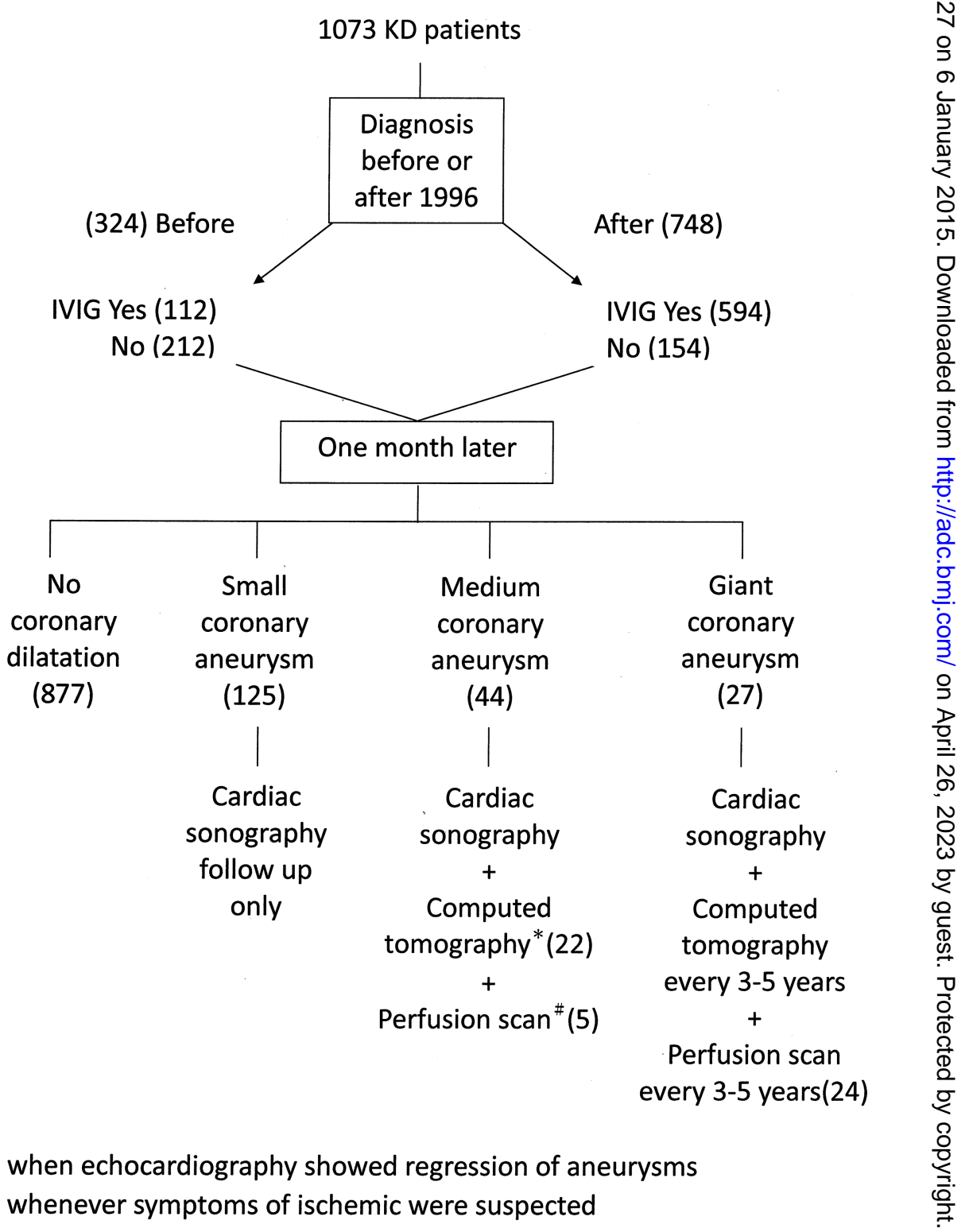


mid-1995 and reimbursed the IVIG only when fever persisted for more than 5 days and (1) more than four presentations of diagnostic criteria were fully filled or (2) there were less than four characteristic criteria but positive coronary involvement. Hence, to avoid the potential selection bias due to the diagnostic era, we stratified our cohort into two strata: early group (1980-1995) and late group (1996-2012). IVIG was given in $34.6 \%(112 / 324)$ and $79.4 \%(594 / 748)$ of the early and late group of patients with $\mathrm{KD}$, respectively $\left(\chi^{2}=200.2, \mathrm{p}<10^{-4}\right.$, figure 1). None received corticosteroids.

CALs occurred in $40.6 \%$ of the patients at their acute febrile stages. Coronary aneurysms, defined as CALs that persisted beyond 1 month of KD onset, were noted in 196 patients (18.3\%, M/F=138/58): small aneurysms in 125 patients $(10.3 \%)$, medium aneurysms in 44 patients $(4.6 \%)$ and giant aneurysms in 27 patients (2.5\%) (table 1, figure 1). Male patient was significantly associated with coronary severity at $\mathrm{KD}$ onset or 1 month later $(\mathrm{p}<0.01)$ in the early and late groups. We failed to demonstrate the association between IVIG resistance and coronary severity $(\mathrm{p}=0.38)$.

In the early group, coronary regression within 1 month after KD onset occurred in 54.1\% (20/37) of those with IVIG and $29.7 \%(19 / 64)$ of those without IVIG $\left(\chi^{2}=7.68, p=0.006\right)$, respectively. In the late group, though the percentage of coronary regression within 1 month was still higher in those with IVIG $(62 \%, 191 / 308)$ than in those without IVIG $(44.8 \%, 13 /$ $29)$, the difference was only borderline due to the small number of patients without IVIG $\left(\chi^{2}=3.28, \mathrm{p}=0.07\right)$.

\section{Long-term outcomes}

The time intervals between $\mathrm{KD}$ onset and the latest follow-up ranged from 1 to 46 (median 5.63) years for all patients, and from 1 to 46 (median 6.67) years for patients with coronary aneurysms. Four deaths occurred and all were male (mortality $0.4 \%$ of patients with $\mathrm{KD}, 2.0 \%$ of patients with coronary aneurysms and $14.8 \%$ of patients with giant aneurysms): one died at 3 years of age because of acute myocardial infarction (AMI), another died at 3 years of age after a heart transplant for ischaemic cardiomyopathy and the last two died suddenly at 16 and 23 years of age, respectively. All four had giant aneurysms. The 10-year and 35-year survival rates of all patients with KD were 0.99 and 0.98 , respectively.

Regarding the late coronary outcomes, among the 877 patients without initial CAL or with transient dilation, none experienced cardiac symptoms or had any positive imaging of CAL during the follow-up (6994 patient-years). Among patients with coronary aneurysms, the coronary aneurysms persisted in $31.4 \%$ of the patients during follow-up. The probability with persistent coronary aneurysm at 5 and 10 years after $\mathrm{KD}$ diagnosis were $27.4 \%$ and $20.6 \%$, respectively (figure $2 \mathrm{~A}$ ). Univariable survival analysis conducted by log-rank test revealed coronary severity 1 month after onset $\left(\chi^{2}=80.73, \mathrm{p}<10^{-3}\right)$ and the era of diagnosis (after
1996, $\left.\chi^{2}=4.99, p=0.025\right)$ was associated with the persistence of coronary aneurysms. Further stratified by the coronary severity 1 month after onset, the factor of diagnosis era (after 1996 vs before 1996) lost its association with aneurysm persistence $\left(\chi^{2}=0.068, \mathrm{p}=0.795\right)$. Myocardial ischaemia, AMI or death occurred in 14 patients, and only one was female $(14 / 196=7.1 \%$; 13 had giant aneurysms and one had a medium aneurysm). All types of ischaemia event-free rates at 10 and 20 years after KD diagnosis were $87.5 \%$ and $73.5 \%$, respectively. Univariable analysis showed two possible risk factors: the severity of the coronary aneurysms 1 month after KD onset $\left(\chi^{2}=33.46, \mathrm{p}<10^{-3}\right)$ and the diagnosis era (after 1996, $\chi^{2}=6.43, p=0.01$ ). Further stratified by the coronary severity 1 month after onset, the factor of diagnosis era lost its association with ischaemia events $\left(\chi^{2}=3.71, p=0.054\right)$.

To avoid the potential confounding effects of coronary severity, we stratified our cohort into three subgroups based on coronary severity 1 month after KD onset and evaluated the roles of IVIG therapy and gender on the late persistence of coronary aneurysms.

Patients with giant aneurysms (27 patients, $M: F=23: 4)$

None of the giant aneurysms regressed during follow-up. In the subgroup of patients with KD with giant aneurysms, the 5-year and 35 -year survival rates were 0.92 and 0.69 , respectively. Except for the four deaths, four additional male patients were diagnosed with AMI, and two of these diagnoses occurred within 1 year of KD onset. Collectively, the incidence of AMI or death in patients with giant aneurysms was $26 \%$, and $67 \%$ of the AMI occurred within the first year of KD onset. Clinical evidence of ischaemia as documented in nuclear medicine studies was also noted in a further five patients (18.5\%), and only one was female. Kaplan-Meier curves for survival free of AMI/death and free of all ischaemia (including ischaemia, myocardial infarction and ischaemia-related death) in patients with $\mathrm{KD}$ with giant aneurysms are shown in figure $3 \mathrm{~A}, \mathrm{~B}$. AMI/death-free survival rates were 0.76 and 0.69 at 10 and 20 years after $K D$ onset, respectively (figure $3 \mathrm{~A}$ ). Ischaemia event-free survival rates were 0.63 and 0.36 at 10 and 20 years, respectively, after disease onset (figure 3B). Men tended to have AMI and ischaemia, and only one ischaemia patient was female. Five-year ischaemia event-free survival rates were 0.68 and 0.75 in patients with giant aneurysms with KD with and without IVIG, respectively. We did log-rank tests but failed to demonstrate an association of all ischaemia with the gender $\left(\chi^{2}=1.01, p=0.32\right)$ or IVIG therapy $\left(\chi^{2}=1.51, \mathrm{p}=0.22\right)$.

Patients with medium aneurysms (44 patients, M:F=34:10)

In those with medium aneurysms, no death occurred and only one patient with persistent coronary aneurysm and stenosis/calcification had clinical evidence of myocardial ischaemia. However, coronary aneurysms persisted in 24 (54.5\%) patients and were associated with stenosis in 8 patients $(14.8 \%)$, and

Table 1 The coronary arterial lesion (CAL) distributions of patients with Kawasaki disease with and without intravenous immunoglobulin (IVIG) therapy

\begin{tabular}{llcccrr}
\hline & Total & CAL in acute phase & CAL 1 month later & Small aneurysm & Medium aneurysm & Giant aneurysm \\
\hline 1980-1995, without IVIG & 212 & 64 & 45 & 17 & 19 & 9 \\
1980-1995, with IVIG & 112 & 37 & 17 & 9 & 6 & 2 \\
1996-2012, with IVIG & 594 & 308 & 117 & 86 & 16 & 15 \\
1996-2012, without IVIG & 154 & 29 & 16 & 13 & 2 & 1 \\
\hline
\end{tabular}



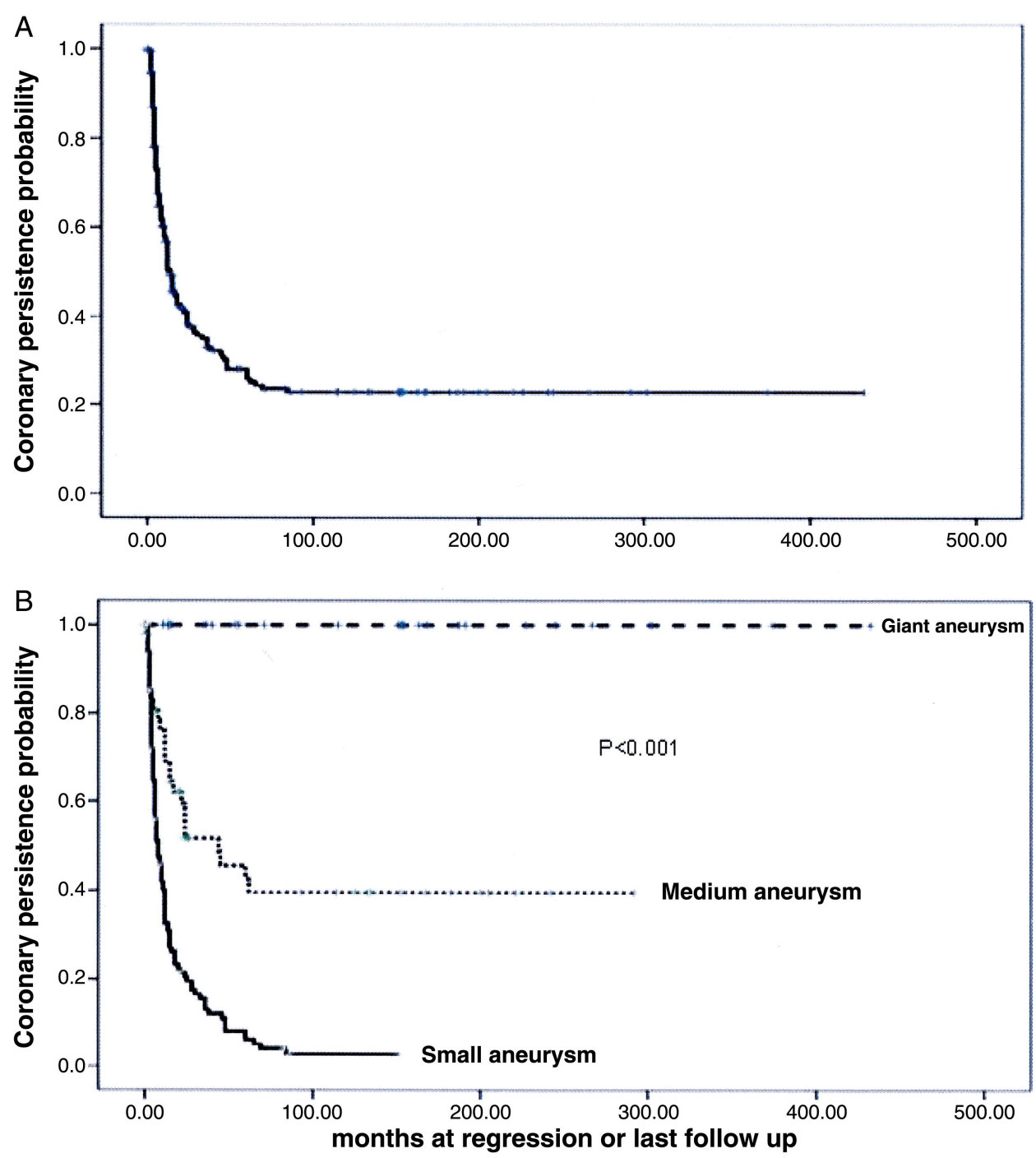

Figure 2 Kaplan-Meier event-free curves showing the probability with persistent coronary arterial aneurysms (A) in those with coronary aneurysms and (B) in the three groups of patients (small, medium and giant coronary aneurysms).

calcification in 4 patients $(9.1 \%)$. The coronary aneurysm persistence probability at 6 months, and at 1,2 and 5 years after $\mathrm{KD}$ onset was $81 \%, 74 \%, 60 \%$ and $46 \%$, respectively (figure 2B). Ten of the 50 patients with medium aneurysms had aneurysms larger than $6 \mathrm{~mm}$. The chances of coronary persistence and the development of stenosis or calcification were similar to patients with aneurysms between 4 and $6 \mathrm{~mm}$. The 5 -year coronary aneurysm persistence probability in patients with IVIG and in those without was 49\% and 39\%, respectively. We did not identify any coronary persistence risk factors in this subgroup (male $\chi^{2}=0.09, p=0.77$; IVIG use $\chi^{2}=0.45, p=0.50$ ).

Patients with small aneurysms (125 patients, M:F=81:44)

In patients with small aneurysms, only one patient reported chest pain that was associated with T-wave inversion without cardiac enzyme elevation. However, the imaging including CT, cardiac catheterisation and nuclear medicine results was all negative, suggesting resolved coronary aneurysm morphology. Coronary aneurysm persisted in 11 patients (8.8\%), mostly in patients with a short follow-up. The probability of small coronary aneurysm persistence at 6 months, and at 1, 2 and 5 years after $\mathrm{KD}$ onset was $55 \%, 31 \%, 20 \%$ and $6 \%$, respectively (figure 2B). The 5-year coronary aneurysm persistence probability in patients with IVIG and in those without were $8.3 \%$ and $5.6 \%$, respectively. No risk factors were identified to predict coronary persistence (IVIG therapy $\chi^{2}=0.19, \mathrm{p}=0.91$; gender $\chi^{2}=0.24, \mathrm{p}=0.63$ ).

To avoid the error of classifying those patients with KD with a naturally dominant right coronary artery system into the small aneurysm subgroup, we further examined the 125 patients with a small aneurysm and found that 10 of them were right coronary artery dominant. The coronary aneurysms of the right coronary artery in these 10 patients regressed to the normal range during follow-up. Therefore, such misclassification did not happen in the current study.

\section{DISCUSSION}

IVIG is the standard therapy for acute KD, and this study demonstrated that, among patients with CALs, coronary regression within 1 month after $\mathrm{KD}$ onset is more likely to be observed in patients with IVIG. However, IVIG use did not further modify the late coronary outcomes, including the aneurysm persistence, AMI/death events and ischaemia. Pathology studies of patients with KD have demonstrated that mononuclear cell infiltration in the coronary vessel walls only predominate within 40 days after KD onset. $^{12} 13$ Thereafter, abundant smooth muscle cells 

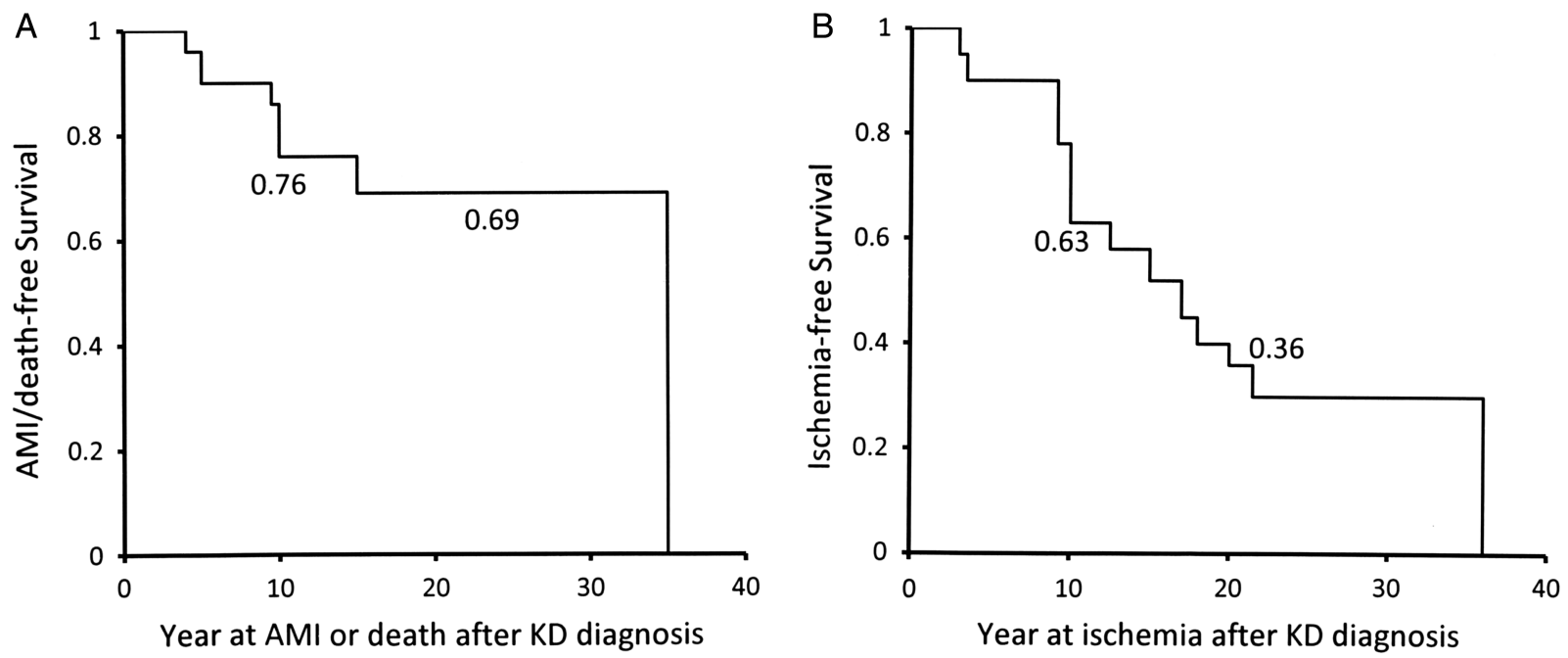

Figure 3 Kaplan-Meier curves for survival free of $\mathrm{AMI} /$ death $(\mathrm{A})$ and all types of ischaemia (myocardial ischaemia, acute myocardial infarction or death) (B) in patients with giant coronary aneurysms (AMI, acute myocardial infarction; KD, Kawasaki disease).

substitute together with vascular remodelling and collagen accumulation. Such clinical and pathology findings suggest that the immunomodulation by IVIG or any other agents should be aggressively delivered as early as possible to improve the coronary severity within 1 month after disease onset.

Our hospital is a tertiary care centre, so referral bias may occur and explain why the incidence of coronary aneurysms $(17.4 \%)$ in this study was higher than those estimated from the Taiwan National Health Insurance database (8\%) and a Japanese nationwide survey $(4-8 \%))^{2}{ }^{7}$ Giant aneurysms were noted in approximately $2.5 \%$ of our patients, which was similar to the incidence rates reported by the Canadian institutes $(1.9-2.2 \%) .{ }^{14}{ }^{15}$ In Japan, the incidence of giant aneurysms was $1.04 \%$ in $1993-1994^{16}$ and decreased to approximately $0.2-0.5 \%$ recently, possibly because of the earlier detection of $\mathrm{KD}$ and aggressive immunomodulation for patients with IVIG resistance, or a bias of the study cohort (institutional vs national cohort). ${ }^{2}$

The long-term prognosis for patients with giant aneurysms is guarded. ${ }^{6}$ In our patients with giant coronary aneurysms, the aneurysms all persisted and one-fourth suffered AMI or death. AMI episodes occurred only in male patients with giant aneurysms. The odds of AMI for men could not be completely explained by the male dominance of $\mathrm{KD}$ occurrence $(\mathrm{M} / \mathrm{F}=1.6)$. In an early report when IVIG therapy was not available, 141 among 195 (72\%) patients with KD with AMI were male. ${ }^{17}$ In another recent report on 60 patients with KD with AMI, 75\% were men. ${ }^{18}$ From our national cohort study, only one patient who experienced AMI following KD was female. ${ }^{7}$ The mechanisms responsible for the unfavourable KD odds for men during adolescence and early adulthood remain unclear. The reason could be that male patient was associated with the formation of giant aneurysms, which are risk factors of coronary ischaemia in patients with KD. Furthermore, in the prospective Coronary Artery Risk Development in Young Adults study on 3043 people aged 18-30 years, the prevalence of coronary arterial calcium in men was much higher than that in women $(15 \%$ vs 5.1\%). ${ }^{19}$ The early adult levels of risk factors were related to subsequent coronary risks. ${ }^{19}$ Therefore, it is possible that male patients with $\mathrm{KD}$, once complicated with coronary giant aneurysms, would also increase the odds of AMI. Aggressive risk factor modification for potential coronary events in high-risk populations is recommended. ${ }^{20}$
The favourable prognosis of small coronary aneurysms has been described. ${ }^{518} 21$ All small aneurysms regressed. However, giant or medium aneurysms clearly add to the cardiovascular risk and such subgroups of patients with KD need more frequent follow-up. In medium aneurysms, we observed that approximately half of the aneurysms persisted, and one-fifth of those with persistent aneurysms exhibited associated stenosis/calcification. However, these patients rarely developed myocardial ischaemia by early adulthood. A previous study demonstrated that at a 10-year follow-up, the probabilities of regression and developing stenosis of the medium coronary aneurysms were $32 \%$ and $20 \%$, respectively. ${ }^{22}$ They also suggested a cut-off threshold for the development of stenosis as $6 \mathrm{~mm}$ for coronary aneurysms, which was not observed in this study.

MRI had been successfully applied to patients with KD for the cardiac evaluation, including the aneurysm, aneurysm, perfusion deficits and scarring. ${ }^{23}$ This would be a better alternative to $\mathrm{CT}$, which is associated with risk from ionising radiation.

\section{Study limitations}

This study was subject to certain limitations. First, this was a retrospective study with a prolonged observation period. Therefore, the chance of early identification and treatment of KD might increase in recent years. The resolution of the echocardiography most likely increases over time, and the proportion of dilations or even small aneurysms might have been underestimated in those diagnosed in an earlier era. Better resolution of echocardiography might also result in increased detection and earlier diagnosis of KD. These would cause important lag time bias. Second, the diagnosis and timely treatment of $\mathrm{KD}$ may also have improved over time, and may be another compounding factor influencing the IVIG therapy widely used after the late 1990s. Third, the current study design was difficult to show the effects from aspirin dosage changed over time. Finally, the number of patients with medium and giant coronary aneurysms was 44 and 27 , respectively, and was relatively small to assess the relationship between the treatment and outcomes.

\section{CONCLUSIONS}

In this longitudinal observational study of patients with $\mathrm{KD}$, we observed that coronary severity 1 month after KD onset is most crucial to the late coronary outcomes, though male patient and 
IVIG therapy were both independent risk factors of initial coronary severity but were not associated with the late coronary outcomes, even in severity stratified subgroups. Aggressive risk modification for patients with $\mathrm{KD}$ with giant and possibly medium coronary aneurysms should be addressed.

Contributors M-TL and L-CS worked on data collection, interpretation and the initial manuscript. E-TW, J-KW and H-CL worked on data interpretation. M-HW had full access to all the data and takes responsibility for the integrity of the data and the accuracy of the data analysis.

Funding This study was supported in part by a grant from the Taiwan National Science Council (NSC 100-2314-B-002-085-MY3).

Competing interests None.

Ethics approval Institutional Review Board of the National Taiwan University Hospital.

Provenance and peer review Not commissioned; externally peer reviewed.

\section{REFERENCES}

1 Kawasaki T. Acute febrile mucocutaneous syndrome with lymphoid involvement with specific desquamation of the fingers and toes in children. Arerugi 1967; 16:178-222.

2 Nakamura $Y$, Yashiro M, Uehara R, et al. Epidemiologic features of Kawasaki disease in Japan: results of the 2005-2006 nationwide survey. J Epidemiol 2008;18:167-72.

3 Eleftheriou D, Levin M, Shingadia D, et al. Management of Kawasaki disease. Arch Dis Child 2014;99:74-83.

4 JCS Joint Working Group. Guidelines for diagnosis and management of cardiovascular sequelae in Kawasaki disease (JCS 2008)—digest version. Circ J 2010;74:1989-2020.

5 Suzuki A, Kamiya T, Tsuda E, et al. Natural history of coronary artery lesions in Kawasaki disease. Prog Pediatr Cardiol 1997;6:211-18.

6 Suda $\mathrm{K}$, lemura $M$, Nishiono $H$, et al. Long-term prognosis of patients with Kawasaki disease complicated by giant coronary aneurysms: a single-institution experience. Circulation 2011;123:1836-42.

$7 \mathrm{Wu}$ MH, Chen HC, Yeh SJ, et al. Prevalence and the long-term coronary risks of patients with Kawasaki disease in a general population $<40$ years: a national database study. Circ Cardiovasc Qual Outcomes 2012;5:566-70

8 Tzen KT, Wu MH, Wang JK, et al. Prognosis of coronary arterial lesions in Kawasak disease treated without intravenous immunoglobulin. Acta Paed Sin 1997;38:32-7.

9 Huang SK, Lin MT, Chen HC, et al. Epidemiology of Kawasaki disease: prevalence from national database and future trends projection by system dynamics modeling. J Pediatr 2013:163:126-31.
10 Newburger JW, Takahashi M, Gerber MA, et al. Committee on Rheumatic Fever, Endocarditis, and Kawasaki Disease, Council on Cardiovascular Disease in the Young, American Heart Association. Diagnosis, treatment, and long-term management of Kawasaki disease: a statement for health professionals from the Committee on Rheumatic Fever, Endocarditis, and Kawasaki Disease, Council on Cardiovascular Disease in the Young. American Heart Association. Pediatrics 2004;114:1708-33.

11 Lin MT, Wang JK, Yeh Jl, et al. Clinical implication of the C allele of the ITPKC gene SNP rs28493229 in Kawasaki disease: association with disease susceptibility and BCG scar reactivation. Pediatr Infect Dis J 2011;30:148-52.

12 Naoe S, Takahashi K, Masuda H, et al. Kawasaki disease with particular emphasis on arterial lesions. Acta Pathol Jpn 1991;41:785-97.

13 Suzuki A, Miyagawa-Tomita S, Nakazawa M, et al. Remodeling of coronary artery lesions due to Kawasaki disease. Jpn Heart J 2000;41:245-56.

14 Levy DM, Silverman ED, Massicotte MP, et al. Longterm outcomes in patients with giant aneurysms secondary to Kawasaki disease. J Rheumatol 2005;32:928-34.

15 McNeal-Davidson A, Fournier A, Scuccimarri R, et al. The fate and observed management of giant coronary artery aneurysms secondary to Kawasaki disease in the Province of Quebec: the complete series since 1976. Pediatr Cardiol 2013;34:170-8.

16 Yanagawa $\mathrm{H}$, Nakamura $\mathrm{Y}$, Yashiro $\mathrm{M}$, et al. Update of the epidemiology of Kawasaki disease in Japan-from the results of 1993-94 nationwide survey. J Epidemiol 1996;6:148-57.

17 Kato $\mathrm{H}$, Ichinose $\mathrm{E}$, Kawasaki T. Myocardial infarction in Kawasaki disease: clinical analyses in 195 cases. J Pediatr 1986;108:923-7.

18 Tsuda E, Hirata T, Matsuo 0, et al. The 30-year outcome for patients after myocardial infarction due to coronary artery lesions caused by Kawasaki disease. Pediatr Cardiol 2011;32:176-82.

19 Loria CM, Liu K, Lewis CE, et al. Early adult risk factor levels and subsequent coronary artery calcification: the CARDIA Study. J Am Coll Cardiol 2007;49:2013-20.

20 Smith SC Jr, Benjamin EJ, Bonow RO, et al. AHA/ACCF secondary prevention and risk reduction therapy for patients with coronary and other atherosclerotic vascular disease: 2011 update: a guideline from the American Heart Association and American College of Cardiology Foundation endorsed by the World Heart Federation and the Preventive Cardiovascular Nurses Association. J Am Coll Cardiol 2011;58:2432-46.

21 Kato $H$, Sugimura T, Akagi T, et al. Long-term consequences of Kawasaki disease. A 10 to 21-year follow-up study of 594 patients. Circulation 1996:94:1379-85.

22 Tsuda E, Kamiya T, Ono Y, et al. Incidence of stenotic lesions predicted by acute phase changes in coronary arterial diameter during Kawasaki disease. Pediatr Cardiol 2005;26:73-9.

23 Mavrogeni S, Papadopoulos G, Hussain T, et al. The emerging role of cardiovascular magnetic resonance in the evaluation of Kawasaki disease. Int J Cardiovasc Imaging 2013;29:1787-98. 